\title{
A NEW FAMILY OF GENERALIZED GAMMA DISTRIBUTION AND ITS APPLICATION
}

\author{
Satsayamon Suksaengrakcharoen and Winai Bodhisuwan
}

Department of Statistics, Faculty of Science, Kasetsart University, Bangkok, 10900, Thailand

Received 2014-03-06; Revised 2014-03-24; Accepted 2014-04-09

\begin{abstract}
The mixture distribution is defined as one of the most important ways to obtain new probability distributions in applied probability and several research areas. According to the previous reason, we have been looking for more flexible alternative to the lifetime data. Therefore, we introduced a new mixed distribution, namely the Mixture Generalized Gamma (MGG) distribution, which is obtained by mixing between generalized gamma distribution and length biased generalized gamma distribution is introduced. The MGG distribution is capable of modeling bathtub-shaped hazard rate, which contains special submodels, namely, the exponential, length biased exponential, generalized gamma, length biased gamma and length biased generalized gamma distributions. We present some useful properties of the MGG distribution such as mean, variance, skewness, kurtosis and hazard rate. Parameter estimations are also implemented using maximum likelihood method. The application of the MGG distribution is illustrated by real data set. The results demonstrate that MGG distribution can provide the fitted values more consistent and flexible framework than a number of distribution include important lifetime data; the generalized gamma, length biased generalized gamma and the three parameters Weibull distributions.
\end{abstract}

Keywords: Generalized Gamma Distribution, Length Biased Generalized Gamma Distribution, Mixture Distribution, Hazard Rate, Life Time Data Analysis

\section{INTRODUCTION}

The family of the gamma distribution is very famous distribution in the literature for analyzing skewed data such as Resti et al. (2013). The Generalized Gamma (GG) distribution was introduced by Stacy (1962) and was included special sub-models such as the exponential, Weibull, gamma and Rayleigh distributions, among other distributions. The GG distribution is appropriated for modeling data with dissimilar types of hazard rate: In the figure of bathtub and unimodal. This typical is practical for estimating individual hazard rate and both relative hazards and relative times by Cox (2008). Its Probability Density Function $(\mathrm{PDF})$ is given by Equation 1 :

$$
g(x)=\frac{\lambda \beta}{\Gamma(\alpha)}(\lambda x)^{\alpha \beta-1} e^{-(\lambda x)^{\beta}} \text { for } x>0 ; \alpha, b, \lambda>0
$$

$$
\begin{aligned}
& \text { Where: } \\
& \begin{aligned}
\alpha \text { and } \beta= & \text { Shape parameters and } \\
\lambda \quad= & \text { A scale parameter } \\
\Gamma(\alpha)= & \text { The gamma function, defined by } \\
& \Gamma(\mathrm{a})=\int_{0}^{\infty} \mathrm{y}^{\mathrm{a}-1} \mathrm{e}^{-\mathrm{y}} \mathrm{dy}
\end{aligned}
\end{aligned}
$$

As well as the Cumulative Distribution Function (CDF) of GG distribution, denoted as $G(x)$, can be expressed as follows Equation 2-4:

$$
G(x)=1-\frac{\Gamma\left(\alpha,(\lambda x)^{\beta}\right)}{\Gamma(\alpha)}
$$

where, $\Gamma(\mathrm{a}, \mathrm{b})=\int_{\mathrm{b}}^{\infty} \mathrm{y}^{\mathrm{a}-1} \mathrm{e}^{-\mathrm{y}} \mathrm{dy}$ is the upper incomplete gamma function.

Corresponding Author: Winai Bodhisuwan, Department of Statistics, Faculty of Science, Kasetsart University, Bangkok, 10900, P.O.Box 1086, Chatuchak, Bangkok, 10903, Thailand Tel: +668 32408592 Fax: +603 89254519 
Furthermore, some useful mathematical properties such as mean and the $r$ th moment are given as follows:

$$
\mathrm{E}_{\mathrm{g}}(\mathrm{x})=\frac{\Gamma\left(\alpha+\frac{1}{\beta}\right)}{\lambda \Gamma(\alpha)}
$$

And:

$$
E_{g}\left(X^{r}\right)=\frac{\Gamma\left(\alpha+\frac{r}{\beta}\right)}{\lambda^{r} \Gamma(\alpha)} \quad r=1,2,3, \ldots
$$

Recently, Ahmed et al. (2013a) presented a Length biased Generalized Gamma (LGG) distribution which obtained pdf as Equation 5:

$$
\mathrm{g}_{\mathrm{L}}(\mathrm{x})=\frac{\lambda \beta}{\Gamma\left(\alpha+\frac{1}{\beta}\right)}(\lambda \mathrm{x})^{\alpha \beta} \mathrm{e}^{-(\lambda x)^{\beta}} \text { for } \mathrm{x}>0 ; \alpha, \beta, \lambda>0
$$

By (5), it is simple to show that the cdf of LGG distribution is given by Equation 6:

$$
\mathrm{G}_{\mathrm{L}}(\mathrm{x})=1-\frac{\Gamma\left(\alpha+\frac{1}{\beta},(\lambda \mathrm{x})^{\beta}\right)}{\Gamma\left(\alpha+\frac{1}{\beta}\right)}
$$

From (5), we can provide some helpful mathematical properties; such as mean and the rth moment of the LGG distribution, respectively are given by Equation 7 and 8:

$$
\mathrm{E}_{\mathrm{L}}(\mathrm{X})=\frac{\Gamma\left(\alpha+\frac{2}{\beta}\right)}{\lambda \Gamma\left(\alpha+\frac{1}{\beta}\right)}
$$

And:

$$
\mathrm{E}_{\mathrm{L}}\left(\mathrm{X}^{\mathrm{r}}\right)=\frac{\Gamma\left(\alpha+\frac{\mathrm{r}+1}{\beta}\right)}{\lambda^{\mathrm{r}} \Gamma\left(\alpha+\frac{1}{\beta}\right)} \mathrm{r}=1,2,3
$$

Moreover, the concept of length biased distribution found in various applications in lifetime area such as family history disease and survival events. The study of human families and wildlife populations were the subject of an article developed by Patil and Rao (1978). Patill et al. (1986) presented a list of the most common forms of the weight function useful in scientific and statistical literature as well as some basic theorems for weighted distributions and length biased as special case they arrived at the conclusion. For example, Nanuwong and Bodhisuwan (2014) presented the length biased Beta Pareto distribution. However, LGG distribution simultaneously provides great flexibility in modeling data in practice. One such class of distributions was generated from the logit of the two-component mixture model, which extends the original family of distributions with the length biased distributions, provide powerful and popular tools for generating flexible distributions with attractive statistical and probabilistic properties.

The mixture distribution is defined as one of the most crucial ways to obtain new probability distributions in applied probability and several research areas. According to the former reason. We have been looking for a more flexible alternative to the Generalized Gamma (GG) distribution. Nadarajah and Gupta (2007) used the GG distribution with application to drought data. Then Cox et al. (2007) offered a parametric survival analysis and taxonomy of the GG distribution. Alkarni (2012) obtained a class of distributions generalizes several distributions with any proper continuous lifetime distribution by compounding truncated logarithmic distribution with decreasing hazard rate. Sattayatham and Talangtam (2012) found the infinite mixture Lognormal distributions for reducing the problem of the number of components and fitting of truncated and/or censored data. Recently, There are many researchers have applied in various field such as Mahesh et al. (2014) proposed a generalized regression neural network for the diagnosis of the hepatitis B virus diease and Biswas et al. (2014) used the networks of the present day communication systems, frequently flood or water logging, sudden failure of one or few nodes in generalized real time multigraphs.

The purpose of this study is to investigate the properties of a new mixture generalized gamma distribution, which was obtained by mixing the GG distribution with the LGG distribution and is more flexible in fitting lifetime data. Section 2 introduces the Mixture Generalized Gamma (MGG) distribution and is concerned with mixture of the GG distribution with the LGG distribution. It contains as well-known lifetime special sub-models. Useful mathematical properties of the MGG distribution including the rth moment, mean, variance, skewness, kurtosis and hazard rate. In addition, section 3 the parameters of the MGG 
distribution are estimated by Maximum Likelihood Estimation (MLE) and are presented the comparison analysis among the GG, LGG, MGG and the three parameters Weibull distributions based on real data set. Finally, conclusion is included in section 4.

\section{MATERIALS AND METHODS}

\subsection{Mixture Generalized Gamma Distribution}

In this section we proposed a new mixture distribution to create extensively flexible distribution and considered some special cases.

\section{Definition 1}

Let $\mathrm{g}(\mathrm{x})$ and $\mathrm{g}_{\mathrm{L}}(\mathrm{x})$ are the pdf and length biased pdf of the random variable (r.v.) $\mathrm{X}$ respectively, where $\mathrm{x}>0$ and $0 \leq p \leq 1$ then the mixture length biased distribution of $X$ produced by the mixture between $g(x)$ and $g_{L}(x)$ in the form of $\mathrm{pg}(\mathrm{x})+(1-\mathrm{p}) \mathrm{g}_{\mathrm{L}}(\mathrm{x})$.

\section{Theorem 1}

Let $\mathrm{X} \sim \operatorname{MGG}(\alpha, \beta, \lambda, \mathrm{p})$. The pdf and cdf respectively are given by Equation 9:

$$
f(x)=\left[\frac{p}{\Gamma(\alpha)}+\frac{(1-p) \lambda x}{\Gamma\left(\alpha+\frac{1}{\beta}\right)}\right] \lambda \beta(\lambda x)^{\alpha \beta-1} e^{-(\lambda x)^{\beta}}
$$

For $\mathrm{x}>0 ; \alpha, \beta, \lambda>0 . ; 0 \leq \mathrm{p} \leq 1$ and Equation 10:

$$
\mathrm{F}(\mathrm{x})=1-\frac{\mathrm{p} \Gamma\left(\alpha,(\lambda \mathrm{x})^{\beta}\right)}{\Gamma(\alpha)}-\frac{(1-\mathrm{p}) \Gamma\left(\alpha+\frac{1}{\beta},(\lambda \mathrm{x})^{\beta}\right)}{\Gamma\left(\alpha+\frac{1}{\beta}\right)}
$$

\section{Proof}

If $\mathrm{X}$ is distributed as MGG distribution with $\alpha, \beta, \lambda$ and mixing $p$ parameters and if its pdf, is obtain by replacement (1) and (5) in Definition 1, (9) called the two-component mixture distribution, can be followed as:

$$
\begin{aligned}
& \mathrm{f}(\mathrm{x})=\mathrm{p}\left[\frac{\lambda \beta}{\Gamma(\alpha)}(\lambda \mathrm{x})^{\alpha \beta-1} \mathrm{e}^{-(\lambda x)^{\beta}}\right]+(1-\mathrm{p}) \\
& {\left[\frac{\lambda \beta}{\Gamma\left(\alpha+\frac{1}{\beta}\right)}(\lambda \mathrm{x})^{\alpha \beta} \mathrm{e}^{-(\lambda x)^{\beta}}\right]=\left[\frac{\mathrm{p}}{\Gamma(\alpha)}+\frac{(1-\mathrm{p}) \lambda \mathrm{x}}{\Gamma\left(\alpha+\frac{1}{\beta}\right)}\right] \lambda \beta(\lambda \mathrm{x})^{\alpha \beta-1} \mathrm{e}^{-(\lambda x)^{\beta}}}
\end{aligned}
$$

Let $F(x)$ is the cdf for a generalized class of distribution for defined by definition 2 , is generated by applying to the MGG distribution Equation 11:

$$
\begin{aligned}
F(x) & =\int_{0}^{x}\left[p g(t)+(1-p) g_{L}(t)\right] d t \\
& =p \int_{0}^{x} g(t) d t+(1-p) \int_{0}^{x} g_{L}(t) d t \\
& =p G(x)+(1-p) G_{L}(x)
\end{aligned}
$$

By substitute (2) and (6) into (11), we then obtain:

$$
\begin{aligned}
\mathrm{F}(\mathrm{x}) & =\mathrm{p}\left[1-\frac{\Gamma(\alpha, \mathrm{x})}{\Gamma(\alpha)}\right]+(1-\mathrm{p})\left[1-\frac{\Gamma\left(\alpha+\frac{1}{\beta}, \mathrm{x}\right)}{\Gamma\left(\alpha+\frac{1}{\beta}\right)}\right] \\
& =1-\frac{\mathrm{p} \Gamma\left(\alpha,(\lambda \mathrm{x})^{\beta}\right)}{\Gamma(\alpha)}-\frac{(1-\mathrm{p}) \Gamma\left(\alpha+\frac{1}{\beta},(\lambda \mathrm{x})^{\beta}\right)}{\Gamma\left(\alpha+\frac{1}{\beta}\right)}
\end{aligned}
$$

In Fig. 1, we present some graphs of MGG distribution, for different values of $\alpha$, similarly in Fig. 2, for $\beta$. We consider some well-known special sub-models of the MGG distribution in the following corollaries.

\section{Corollary 1}

If $p=0$ then the MGG distribution reduces to the LGG distribution with parameters $\alpha, \beta$ and $\lambda$ is defined by Equation 12:

$$
f(x)=\frac{\lambda \beta}{\Gamma\left(\alpha+\frac{1}{\beta}\right)}(\lambda x)^{\alpha \beta} e^{-(\lambda x)^{\beta}}
$$

\section{Proof}

Substituting $\mathrm{p}=0$ into (9), we obtained (12) which is introduced by Ahmed et al. (2013b).

\section{Corollary 2}

If $\alpha=\beta=1$ and $p=0$, then the MGG distribution deduces to length biased exponential distribution Ahmed et al. (2013a) and its pdf is given by:

$$
f(x)=\lambda^{2} x e^{-\lambda x}
$$




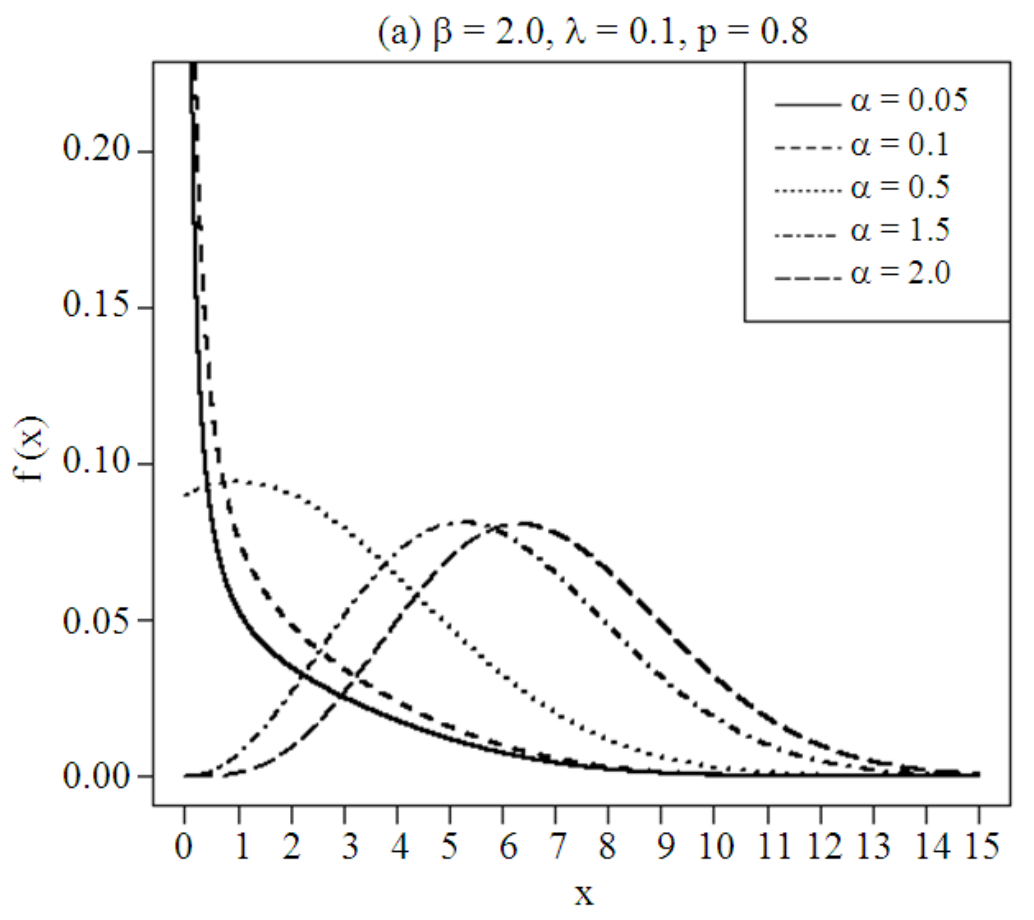

Fig. 1. The pdf of MGG distribution for different values of $\alpha$

(b) $\alpha=2.0, \lambda=0.1, p=0.4$

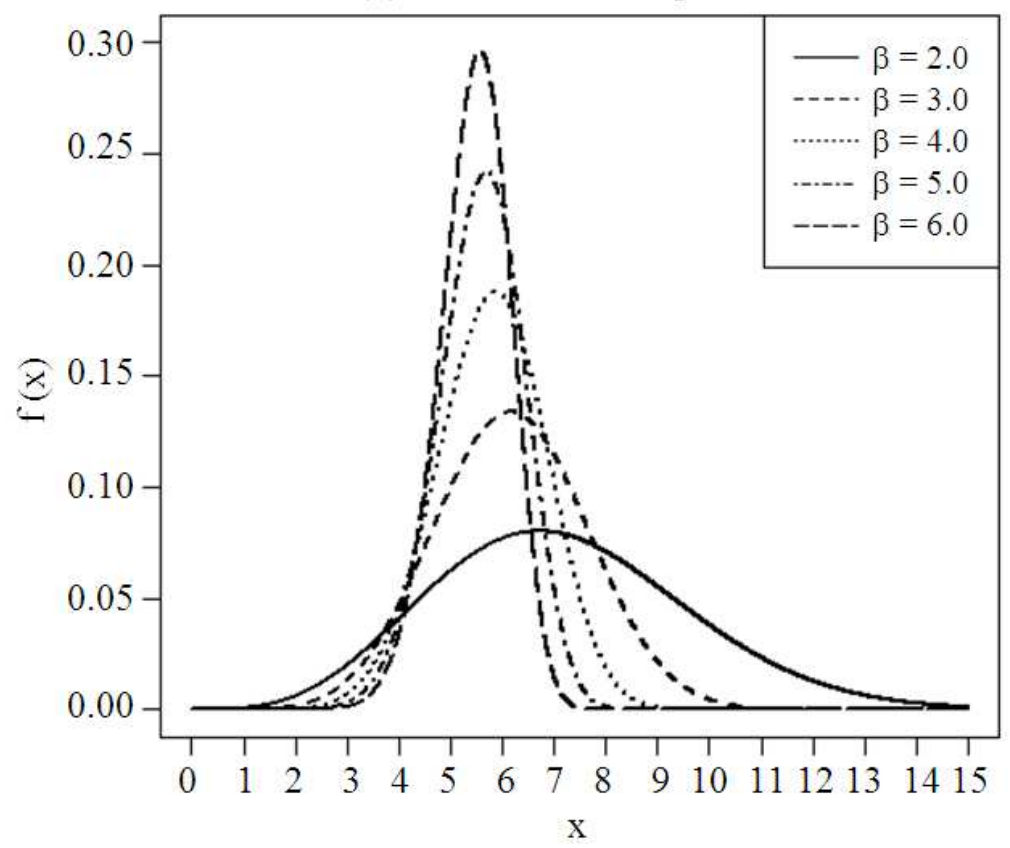

Fig. 2. The pdf of MGG distribution for different values of $\beta$ 


\section{Proof}

Substituting $\alpha=\beta=1$ into (12) reduces to

$$
f(x)=\lambda^{2} x e^{-\lambda x}
$$

\section{Corollary 3}

If $\beta=1$ and $p=0$ then the MGG distribution reduces to length biased gamma distribution which presented by Ahmed et al. (2013b) as follows:

$$
f(x)=\frac{\lambda^{\alpha+1}}{\Gamma(\alpha+1)} x^{\alpha} e^{-\lambda x}
$$

\section{Proof}

Replacing $\beta=1$ in (12), we have:

$$
f(x)=\frac{\lambda^{\alpha+1}}{\Gamma(\alpha+1)} x^{\alpha} e^{-\lambda x}
$$

\section{Corollary 4}

If $\mathrm{p}=1$, then the MGG distribution derived to $\mathrm{GG}$ distribution and its pdf is defined by Stacy (1962) Equation 13:

$$
f(x)=\frac{\lambda \beta}{\Gamma(\alpha)}(\lambda x)^{\alpha \beta-1} e^{-(\lambda x)^{\beta}}
$$

\section{Proof}

Replacing $\mathrm{p}=1$ in (9) may be expressed as (1).

\section{Corollary 5}

If $\alpha=\beta=1$ and $p=1$, then the MGG distribution reduces to exponential distribution and its pdf can be written as:

$$
f(x)=\lambda e^{-\lambda x}
$$

\section{Proof}

Replacing $\alpha=\beta=1$ in (13) we obtain:

$$
f(x)=\lambda e^{-\lambda x}
$$

\subsection{Moments of the MGG Distribution}

In this section, we will consider the rth moment of r.v. $\mathrm{X} \sim \mathrm{MGG}(\alpha, \beta, \lambda, \mathrm{p})$. The MGG distribution presents various properties including: The rth moment, mean, variance, coefficient of kurtosis, coefficient of skewness and hazard rate are provided as follows:

\section{Definition 2}

$E_{g}\left(X^{r}\right)$ and $E_{L}\left(X^{r}\right)$ are the rth moments of original distribution and length biased distribution of the r.v. $X$ respectively. If $0 \leq p \leq 1$, then the rth moments of the mixture distribution is define by:

$$
\begin{aligned}
& E\left(X^{r}\right)=p E_{g}\left(X^{r}\right)+(1-p) E_{L}^{r}(X) \\
& x>0, r=1,2,3
\end{aligned}
$$

\section{Theorem 2}

Let $\mathrm{X} \sim \operatorname{MGG}(\alpha, \beta, \lambda, p)$, the rth moment of r.v. $\mathrm{X}$ is written Equation 14:

$\mathrm{E}\left(\mathrm{X}^{\mathrm{r}}\right)=\frac{1}{\lambda^{\mathrm{r}}}\left[\frac{\mathrm{p} \Gamma\left(\alpha+\frac{\mathrm{r}}{\beta}\right)}{\Gamma(\alpha)}+\frac{(1-\mathrm{p}) \Gamma\left(\alpha+\frac{\mathrm{r}+1}{\beta}\right)}{\Gamma\left(\alpha+\frac{1}{\beta}\right)}\right]$

where, $x>0, r=1,2,3, \ldots, 0 \leq p \leq 1$.

\section{Proof}

If $\mathrm{X} \sim \mathrm{MGG}(\alpha, \beta, \lambda, \mathrm{p})$ from Definition 2 , by substitute (4) and (8), then the rth moment is given by:

$$
\begin{aligned}
\mathrm{E}\left(\mathrm{X}^{\mathrm{r}}\right) & =\mathrm{p}\left[\frac{\Gamma\left(\alpha+\frac{\mathrm{r}}{\beta}\right)}{\lambda^{\mathrm{r}} \Gamma(\alpha)}\right]+(1-\mathrm{p})\left[\frac{\Gamma\left(\alpha+\frac{\mathrm{r}+1}{\beta}\right)}{\lambda^{\mathrm{r}} \Gamma\left(\alpha+\frac{1}{\beta}\right)}\right] \\
& =\frac{1}{\lambda^{\mathrm{r}}}\left[\frac{\mathrm{p} \Gamma\left(\alpha+\frac{\mathrm{r}}{\beta}\right)}{\Gamma(\alpha)}+\frac{(1-\mathrm{p}) \Gamma\left(\alpha+\frac{\mathrm{r}+1}{\beta}\right)}{\Gamma\left(\alpha+\frac{1}{\beta}\right)}\right]
\end{aligned}
$$

From (14), it is straightforward to mean, the second four moments and variance respectively as:

$$
\begin{aligned}
& \mathrm{E}(\mathrm{X})=\frac{1}{\lambda}\left[\frac{\mathrm{p} \Gamma\left(\alpha+\frac{1}{\beta}\right)}{\Gamma(\alpha)}+\frac{(1-\mathrm{p}) \Gamma\left(\alpha+\frac{2}{\beta}\right)}{\Gamma\left(\alpha+\frac{1}{\beta}\right)}\right] \\
& \mathrm{E}\left(\mathrm{X}^{2}\right)=\frac{1}{\lambda^{2}}\left[\frac{\mathrm{p} \Gamma\left(\alpha+\frac{2}{\beta}\right)}{\Gamma(\alpha)}+\frac{(1-\mathrm{p}) \Gamma\left(\alpha+\frac{3}{\beta}\right)}{\Gamma\left(\alpha+\frac{1}{\beta}\right)}\right]
\end{aligned}
$$




$$
\begin{array}{r}
\mathrm{E}\left(\mathrm{X}^{3}\right)=\frac{1}{\lambda^{3}}\left[\frac{\mathrm{p} \Gamma\left(\alpha+\frac{3}{\beta}\right)}{\Gamma(\alpha)}+\frac{(1-\mathrm{p}) \Gamma\left(\alpha+\frac{4}{\beta}\right)}{\Gamma\left(\alpha+\frac{1}{\beta}\right)}\right] \\
\mathrm{E}\left(\mathrm{X}^{4}\right)=\frac{1}{\lambda^{4}}\left[\frac{\mathrm{p} \Gamma\left(\alpha+\frac{4}{\beta}\right)}{\Gamma(\alpha)}+\frac{(1-\mathrm{p}) \Gamma\left(\alpha+\frac{5}{\beta}\right)}{\Gamma\left(\alpha+\frac{1}{\beta}\right)}\right] \\
\operatorname{Var}(\mathrm{X})=\frac{1}{\lambda^{2}}\left[\frac{\mathrm{p} \Gamma\left(\alpha+\frac{2}{\beta}\right)}{\Gamma(\alpha)}+\frac{(1-\mathrm{p}) \Gamma\left(\alpha+\frac{3}{\beta}\right)}{\Gamma\left(\alpha+\frac{1}{\beta}\right)}\right] \\
\left.-\left[\frac{\mathrm{p} \Gamma\left(\alpha+\frac{1}{\beta}\right)}{\Gamma(\alpha)}+\frac{(1-\mathrm{p}) \Gamma\left(\alpha+\frac{2}{\beta}\right)}{\Gamma\left(\alpha+\frac{1}{\beta}\right)}\right]\right\}
\end{array}
$$

We set:

$$
\omega(\alpha, \beta, \mathrm{p}, \mathrm{i})=\frac{\mathrm{p} \Gamma\left(\alpha+\frac{1}{\beta}\right) \Gamma\left(\alpha+\frac{\mathrm{i}}{\beta}\right)+(1-\mathrm{p}) \Gamma(\alpha) \Gamma\left(\alpha+\frac{\mathrm{i}+1}{\beta}\right)}{\Gamma(\alpha) \Gamma\left(\alpha+\frac{1}{\beta}\right)}
$$

Note that, $\omega(\alpha, \beta, \mathrm{p}, \mathrm{i})$ is defined when $\mathrm{i} \in \mathrm{I}^{+}$and let, $\mathrm{W}=\sqrt{\omega(\alpha, \beta, \mathrm{p}, 2)-\omega^{2}(\alpha, \beta, \mathrm{p}, 1)}$ consequently, the coefficient of skewness $\left(\alpha_{3}\right)$ in (15) and the coefficient of kurtosis $\left(\alpha_{4}\right)$ in (16) can be written as Equation 15 and 16:

$$
\begin{aligned}
& \alpha_{3}= \\
& \frac{\left[\omega(\alpha, \beta, p, 3)-3 \omega(\alpha, \beta, p, 2) \omega(\alpha, \beta, p, 1)+2 \omega^{3}(\alpha, \beta, p, 1)\right]}{\mathrm{W}^{3}} \\
& \alpha_{4}=[\omega(\alpha, \beta, \mathrm{p}, 4)-4 \omega(\alpha, \beta, \mathrm{p}, 3) \omega(\alpha, \beta, \mathrm{p}, 1) \\
& \left.\quad+6 \omega(\alpha, \beta, \mathrm{p}, 2) \omega^{2}(\alpha, \beta, \mathrm{p}, 1)-3 \omega^{4}(\alpha, \beta, \mathrm{p}, 1)\right] / \mathrm{W}^{4}
\end{aligned}
$$

We illustrate activities of mean and variance in Table 1 that are increasing functions of $\alpha$. Also, Table 2 show skewness in (15) and kurtosis in (16) for different values $\alpha$ and $p$ are independent of parameter $\alpha$. Moreover, we discover that both the skewness and kurtosis are increasing functions of $p$ except are both decreasing functions of $\alpha$.

\subsection{Hazard Rate}

Hazard rate (or failure rate) are expansively apply in several fields. For example; Wahyudi et al. (2011) offered the trivariate hazard rate function of trivariate liftime distribution. By definition, the hazard rate of a r.v. $X$ with $\operatorname{pdf} f(x)$ and $\operatorname{cdf} F(x)$ can be written by:

$$
h(x)=\frac{f(x)}{1-F(x)}
$$

Using (9) and (10), the hazard rate of the MGG distribution may be expressed as Equation 17:

$$
\begin{aligned}
\mathrm{h}(\mathrm{x}) & =\frac{\left[\frac{\mathrm{p}}{\Gamma(\alpha)}+\frac{(1-\mathrm{p}) \lambda \mathrm{x}}{\Gamma\left(\alpha+\frac{1}{\beta}\right)}\right] \lambda \beta(\lambda \mathrm{x})^{\alpha \beta-1} \mathrm{e}^{-(\lambda x)^{\beta}}}{1-\left\{1-\frac{\mathrm{p} \Gamma(\alpha, \mathrm{x})}{\Gamma(\alpha)}-(1-\mathrm{p}) \frac{\Gamma\left(\alpha+\frac{1}{\beta}, \mathrm{x}\right)}{\Gamma\left(\alpha+\frac{1}{\beta}\right)}\right\}} \\
= & \frac{\left[\mathrm{p} \Gamma\left(\alpha+\frac{1}{\beta}\right)+(1-\mathrm{p}) \lambda \mathrm{x} \Gamma(\alpha)\right] \lambda \beta(\lambda \mathrm{x})^{\alpha \beta-1} \mathrm{e}^{-(\lambda x)^{\beta}}}{\mathrm{p} \Gamma(\alpha, \mathrm{x}) \Gamma\left(\alpha+\frac{1}{\beta}\right)+(1-\mathrm{p}) \Gamma(\alpha) \Gamma\left(\alpha+\frac{1}{\beta}, \mathrm{x}\right)}
\end{aligned}
$$

When substituting different values of parameters in (17) then we get some hazard rate of the MGG distribution which it present in Fig. 3:

- When $p=0$ then the hazard rate of the MGG distribution reduces to the hazard rate of the LGG distribution

- When $p=1$ then the hazard rate of the MGG distribution deduces to the hazard rate of the GG distribution

- When $\alpha=\beta=p=1$ then the hazard rate of the MGG distribution derived to the hazard rate of the exponential distribution

\subsection{Limit Behaviour}

The limit of pdf of MGG as $x \rightarrow \infty$ is 0 and the limit as $x \rightarrow 1 / \lambda$ is given by: 


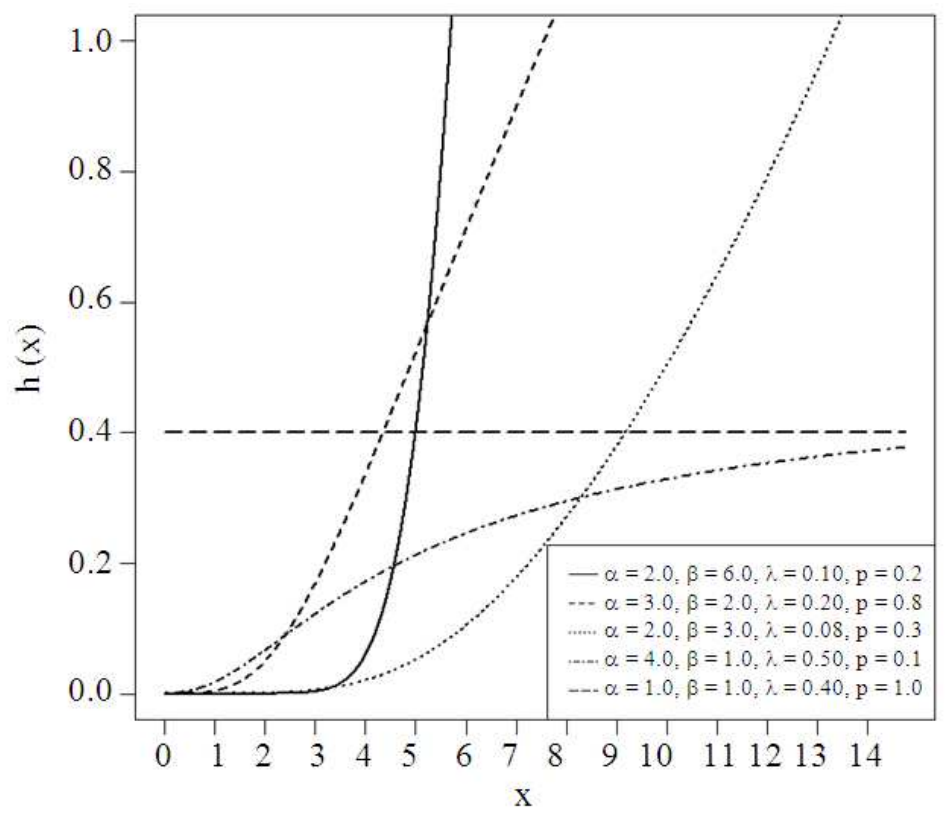

Fig. 3. Plot of the hazard rates of the MGG distribution for different values of parameters

Table 1. Mean and variance of MGG distribution for various values of $\alpha, \beta, \lambda$ and $p$

\begin{tabular}{|c|c|c|c|c|c|c|c|c|}
\hline \multirow[b]{2}{*}{$\alpha$} & \multirow[b]{2}{*}{$\beta$} & \multirow[b]{2}{*}{$\lambda$} & \multicolumn{2}{|l|}{$\mathrm{p}=0.2$} & \multicolumn{2}{|l|}{$\mathrm{p}=0.5$} & \multicolumn{2}{|l|}{$\mathrm{p}=0.8$} \\
\hline & & & Mean & Variance & Mean & Variance & Mean & Variance \\
\hline \multirow[t]{9}{*}{$\overline{3}$} & 2 & 0.1 & 17.7666 & 24.3478 & 17.3354 & 24.4835 & 16.9042 & 24.2474 \\
\hline & & 0.3 & 5.9222 & 2.7053 & 5.77850 & 2.7204 & 5.6347 & 2.6942 \\
\hline & & 0.9 & 1.9741 & 0.3006 & 1.92620 & 0.3023 & 1.8782 & 0.2994 \\
\hline & 4 & 0.1 & 12.9785 & 3.5930 & 12.8914 & 3.6457 & 12.8043 & 3.6832 \\
\hline & & 0.3 & 4.3262 & 0.3992 & 4.29710 & 0.4051 & 4.2681 & 0.4092 \\
\hline & & 0.9 & 1.4421 & 0.0444 & 1.43240 & 0.0450 & 1.4227 & 0.0455 \\
\hline & 6 & 0.1 & 11.8225 & 1.3829 & 11.7861 & 1.4006 & 11.7496 & 1.4157 \\
\hline & & 0.3 & 3.9408 & 0.1537 & 3.92870 & 0.1556 & 3.9165 & 0.1573 \\
\hline & & 0.9 & 1.3136 & 0.0171 & 1.30960 & 0.0173 & 1.3055 & 0.0175 \\
\hline \multirow[t]{9}{*}{5} & 2 & 0.1 & 22.7025 & 24.5948 & 22.3676 & 24.6885 & 22.0328 & 24.5578 \\
\hline & & 0.3 & 7.5675 & 2.7328 & 7.45590 & 2.7432 & 7.3443 & 2.7286 \\
\hline & & 0.9 & 2.5225 & 0.3036 & 2.48530 & 0.3048 & 2.4481 & 0.3032 \\
\hline & 4 & 0.1 & 14.8265 & 2.7915 & 14.7684 & 2.8160 & 14.7102 & 2.8338 \\
\hline & & 0.3 & 4.9422 & 0.3102 & 4.92280 & 0.3129 & 4.9034 & 0.3149 \\
\hline & & 0.9 & 1.6474 & 0.0345 & 1.64090 & 0.0348 & 1.6345 & 0.0350 \\
\hline & 6 & 0.1 & 12.9538 & 0.9717 & 12.9308 & 0.9790 & 12.9078 & 0.9852 \\
\hline & & 0.3 & 4.3179 & 0.1080 & 4.31030 & 0.1088 & 4.3026 & 0.1095 \\
\hline & & 0.9 & 1.4393 & 0.0120 & 1.43680 & 0.0121 & 1.4342 & 0.0122 \\
\hline \multirow[t]{9}{*}{8} & 2 & 0.1 & 28.5527 & 24.7420 & 28.2877 & 24.8049 & 28.0227 & 24.7274 \\
\hline & & 0.3 & 9.5176 & 2.7491 & 9.42920 & 2.7561 & $\begin{array}{r}20.021 \\
9.3409\end{array}$ & 2.7475 \\
\hline & & 0.9 & 3.1725 & 0.3055 & 3.14310 & 0.3062 & 3.1136 & 0.3053 \\
\hline & 4 & 0.1 & 16.7276 & 2.2090 & 16.6873 & 2.2211 & 16.6469 & 2.2299 \\
\hline & & 0.3 & 5.5759 & 0.2454 & 5.56240 & 0.2468 & 5.549 & 0.2478 \\
\hline & & 0.9 & 1.8586 & 0.0273 & 1.85410 & 0.0274 & 1.8497 & 0.0275 \\
\hline & 6 & 0.1 & 14.0589 & 0.7047 & 14.0437 & 0.7079 & 14.0284 & 0.7107 \\
\hline & & 0.3 & 4.6863 & 0.0783 & 4.68120 & 0.0787 & 4.6761 & 0.0790 \\
\hline & & 0.9 & 1.5621 & 0.0087 & 1.56040 & 0.0087 & 1.5587 & 0.0088 \\
\hline
\end{tabular}


Table 2. Skewness and kurtosis of MGG distribution for various values of $\alpha, \beta$ and $p$

\begin{tabular}{|c|c|c|c|c|c|c|c|}
\hline \multirow[b]{2}{*}{$\alpha$} & \multirow[b]{2}{*}{$\beta$} & \multicolumn{2}{|l|}{$\mathrm{p}=0.2$} & \multicolumn{2}{|l|}{$\mathrm{p}=0.5$} & \multicolumn{2}{|l|}{$\mathrm{p}=0.8$} \\
\hline & & Skewness & Kurtosis & Skewness & Kurtosis & Skewness & Kurtosis \\
\hline \multirow[t]{3}{*}{3} & 1.0 & 1.0132 & 4.5298 & 1.0672 & 4.6800 & 1.1327 & 4.9082 \\
\hline & 1.5 & 0.5367 & 3.3260 & 0.5554 & 3.3444 & 0.5814 & 3.3833 \\
\hline & 2.0 & 0.2886 & 3.0143 & 0.2961 & 3.0131 & 0.3094 & 3.0190 \\
\hline \multirow[t]{3}{*}{5} & 1.0 & 0.8236 & 4.0132 & 0.8522 & 4.0775 & 0.8845 & 4.1675 \\
\hline & 1.5 & 0.4277 & 3.2058 & 0.4368 & 3.2127 & 0.4494 & 3.2272 \\
\hline & 2.0 & 0.2241 & 3.0055 & 0.2274 & 3.0047 & 0.2335 & 3.0066 \\
\hline \multirow[t]{3}{*}{8} & 1.0 & 0.6705 & 3.6725 & 0.6858 & 3.7004 & 0.7023 & 3.7375 \\
\hline & 1.5 & 0.3437 & 3.1324 & 0.3483 & 3.1351 & 0.3547 & 3.1409 \\
\hline & 2.0 & 0.1771 & 3.0022 & 0.1787 & 3.0019 & 0.1817 & 3.0025 \\
\hline
\end{tabular}

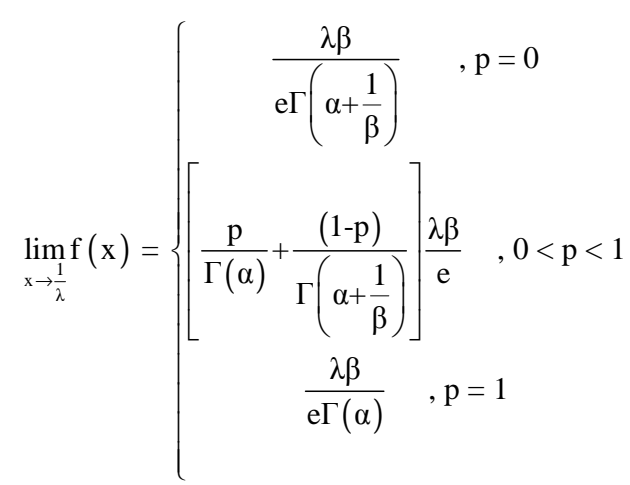

It is straightforward to demonstrate the above from the pdf of MGG in (9) as:

$$
\begin{aligned}
\lim _{x \rightarrow \frac{1}{\lambda}} f(x) & =\lim _{x \rightarrow \frac{1}{\lambda}}\left\{\left[\frac{\mathrm{p}}{\Gamma(\alpha)}+\frac{(1-\mathrm{p}) \lambda \mathrm{x}}{\Gamma\left(\alpha+\frac{1}{\beta}\right)}\right] \lambda \beta(\lambda \mathrm{x})^{\alpha \beta-1} \mathrm{e}^{-(\lambda x)^{\beta}}\right\} \\
& =\left[\frac{\mathrm{p}}{\Gamma(\alpha)}+\frac{(1-\mathrm{p})}{\Gamma\left(\alpha+\frac{1}{\beta}\right)}\right] \frac{\lambda \beta}{\mathrm{e}} .
\end{aligned}
$$

\section{RESULTS}

\subsection{Parameters Estimation}

The estimation of parameters for the MGG distribution will be discussed via the MLE method procedure. The likelihood function of the $\operatorname{MGG}(\alpha, \beta, \lambda$, p) is given by:

$$
\mathrm{L}(\mathrm{x} ; \theta)=\prod_{\mathrm{i}=1}^{\mathrm{n}}\left\{\left[\frac{\mathrm{p}}{\Gamma(\alpha)}+\frac{(1-\mathrm{p}) \lambda \mathrm{x}_{\mathrm{i}}}{\Gamma\left(\alpha+\frac{1}{\beta}\right)}\right] \lambda \beta\left(\lambda \mathrm{x}_{\mathrm{i}}\right)^{\alpha \beta-1} \mathrm{e}^{-\left(\lambda \mathrm{x}_{\mathrm{i}}\right)^{\beta}}\right\}
$$

From which we calculate approximately the loglikelihood function Equation 18:

$$
\begin{aligned}
\log L(\theta)= & \operatorname{nlog}(\lambda \beta)+(\alpha \beta-1) \sum_{\mathrm{i}=1}^{\mathrm{n}} \log \left(\lambda \mathrm{x}_{\mathrm{i}}\right)-\lambda^{\beta} \sum_{\mathrm{i}=1}^{\mathrm{n}} \mathrm{x}_{\mathrm{i}}^{\beta} \\
& +\sum_{\mathrm{i}=1}^{\mathrm{n}} \log \left[\frac{\mathrm{p}}{\Gamma(\alpha)}+\frac{(1-\mathrm{p}) \lambda \mathrm{x}_{\mathrm{i}}}{\Gamma\left(\alpha+\frac{1}{\beta}\right)}\right]
\end{aligned}
$$

The first order conditions for finding the optimal values of the parameters were obtained by differentiating (18) with respect to $\alpha, \beta, \lambda$ and $p$ we get the following differential Equation 19-22:

$$
\begin{aligned}
& \frac{\partial}{\partial \alpha} \log L(\theta)=\sum_{\mathrm{i}=1}^{\mathrm{n}} \log \left(\lambda \mathrm{x}_{\mathrm{i}}\right) \\
& -\sum_{\mathrm{i}=1}^{\mathrm{n}}\left\{\frac{\mathrm{p} \Gamma^{\prime}(\alpha) \Gamma^{2}\left(\alpha+\frac{1}{\beta}\right)+(1-\mathrm{p}) \lambda \mathrm{x}_{\mathrm{i}} \Gamma^{2}(\alpha) \Gamma^{\prime}\left(\alpha+\frac{1}{\beta}\right)}{\Gamma(\alpha) \Gamma\left(\alpha+\frac{1}{\beta}\right)\left[\mathrm{p} \Gamma\left(\alpha+\frac{1}{\beta}\right)+(1-\mathrm{p}) \lambda \mathrm{x}_{\mathrm{i}} \Gamma(\alpha)\right]}\right\} \\
& \frac{\partial}{\partial \beta} \operatorname{logL}(\theta)=\frac{\mathrm{n}}{\beta}+\sum_{\mathrm{i}=1}^{\mathrm{n}} \log \left(\lambda \mathrm{x}_{\mathrm{i}}\right)-\lambda^{\beta+1} \sum_{\mathrm{i}=1}^{\mathrm{n}}\left(\mathrm{x}_{\mathrm{i}} \log \mathrm{x}_{\mathrm{i}}\right) \\
& -\sum_{\mathrm{i}=1}^{\mathrm{n}}\left\{\frac{(1-\mathrm{p}) \lambda \mathrm{x}_{\mathrm{i}} \Gamma(\alpha) \Gamma^{\prime}\left(\alpha+\frac{1}{\beta}\right)}{(1-\mathrm{p}) \lambda \mathrm{x}_{\mathrm{i}} \Gamma(\alpha) \Gamma\left(\alpha+\frac{1}{\beta}\right)+\mathrm{p} \Gamma^{2}\left(\alpha+\frac{1}{\beta}\right)}\right\}
\end{aligned}
$$


Table 3. Maximum likelihood estimates and K-S distances with their associated p-values for the four mixture distributions fitted to depressive condition data

\begin{tabular}{|c|c|c|c|}
\hline Distributions & Maximum likelihood estimates & K-S statistic & p-value \\
\hline \multirow[t]{2}{*}{ MGGD } & $\hat{\alpha}=11.8383, \hat{\beta}=0.2832$ & & \\
\hline & $\hat{\lambda}=1.8115, \hat{\mathrm{p}}=0.7377$ & 0.0941 & 0.8484 \\
\hline \multirow[t]{2}{*}{ LGGD } & $\hat{\alpha}=0.5908, \hat{\beta}=0.5758$, & & \\
\hline & $\hat{\lambda}=0.0008$ & 0.1041 & 0.7535 \\
\hline \multirow[t]{2}{*}{ GGD } & $\hat{\alpha}=0.9999, \hat{\beta}=1.0025$, & & \\
\hline & $\hat{\lambda}=0.0001$ & 0.1505 & 0.3083 \\
\hline \multirow[t]{2}{*}{ Weibull } & $\hat{\alpha}=318, \hat{\beta}=0.7032$, & & \\
\hline & $\hat{\lambda}=5127.8$ & 0.1660 & 0.2081 \\
\hline
\end{tabular}

$\frac{\partial}{\partial \lambda} \log L(\theta)=\frac{n}{\lambda}+(\alpha \beta-1) \sum_{i=1}^{n} \log x_{i}-\beta \lambda^{\beta-1} \sum_{i=1}^{n} x_{i}^{\beta}$

$+\sum_{\mathrm{i}=1}^{\mathrm{n}}\left[\frac{(1-\mathrm{p}) \mathrm{x}_{\mathrm{i}} \Gamma(\alpha) \Gamma\left(\alpha+\frac{1}{\beta}\right)}{\mathrm{p} \Gamma^{2}\left(\alpha+\frac{1}{\beta}\right)+(1-\mathrm{p}) \lambda \mathrm{x}_{\mathrm{i}} \Gamma(\alpha)}\right]$

And:

$$
\frac{\partial}{\partial \mathrm{p}} \log \mathrm{L}(\theta)=\sum_{\mathrm{i}=1}^{\mathrm{n}}\left[\frac{\Gamma\left(\alpha+\frac{1}{\beta}\right)-\lambda \mathrm{x}_{\mathrm{i}} \Gamma(\alpha)}{(1-\mathrm{p}) \lambda \mathrm{x}_{\mathrm{i}} \Gamma(\alpha)+\mathrm{p} \Gamma\left(\alpha+\frac{1}{\beta}\right)}\right]
$$

These four derivative equations cannot be solved analytically, as they need to rely on Newton-Raphson: The Newton-Raphson method is a powerful technique for solving equations numerically. In practice $\hat{\alpha}, \hat{\beta}, \hat{\lambda}$ and $\hat{p}$ are the solution of the estimating equations obtained by differentiating the likelihood in terms of $\alpha, \beta, \lambda$ and $\mathrm{p}$ solving in (19)-(22) to zero. Therefore, $\hat{\alpha}, \hat{\beta}, \hat{\lambda}$ and $\hat{\mathrm{p}}$ can be obtained by solving the resulting equations simultaneously using a numerical procedure with the Newton-Raphson method.

\subsection{Applications of the MGG Distribution}

For one application of the MGG distribution, we used a real data set. This was the flood rates data from the Floyd River located in James, Iowa, USA for the years 1935-1973 from Akinsete et al. (2008). The maximum likelihood method provides parameters estimation. By comparing these fitting distribution in Table 3 based on the p-value of this comparison, the results have shown that the MGG distribution provided a better fit than the GG, LGG and the three parameters Weibull distributions. Since, Mahdi and Gupta (2013) presented the three parameters Weibull distribution obtained the pdf as:

$$
\mathrm{f}_{\mathrm{w}}(\mathrm{x})=\frac{\beta}{\lambda}\left(\frac{\mathrm{x}-\alpha}{\lambda}\right)^{\beta-1} \mathrm{e}^{-\left(\frac{\mathrm{x}-\alpha}{\lambda}\right)^{\beta}} \text { for } \mathrm{x}>0 ; \alpha, \beta, \lambda>0
$$

\section{DISCUSSION}

The MGG distribution is significance of mixture distribution method which is a new family of GG distribution. In this study, the MGG distribution found that it provides a considerably better fit than the LGG and GG distributions which are some sub-models of the MGG distribution. Indicating that MGG distribution makes the approach moderately useful for lifetime data. Based on p-values of the MGG distribution is better than LGG, GG and three parameters Weibull distributions. As well as, the research by Kamaruzzaman et al. (2012) fit the two component mixture normal distribution by using data sets on logarithmic stock returns of Bursa, Malaysia indices better than a normal distribution. Furthemore, Cordeiro et al. (2012) suggested the Kumaraswamy generalized half-normal distribution using the flood rates data of the Floyd River, located in James, Iowa, USA provides a better fit than sub-models of it. In addition, Faton and Llukan 2014 generalize the Pareto distribution can be used quite effectively to provide better fits than the Pareto distribution.

\section{CONCLUSION}

This study offers the MGG distribution which is obtained by mixing GG distribution with LGG 
distribution. We showed that the LGG, GG, Gamma, length biased exponential and exponential distributions are sub-models of this new mixed distribution. We have derived several properties of the MGG distribution which includes mean, variance, skewness, kurtosis and hazard rate. Additionally, parameters estimation are also implemented using MLE method and the usefulness of this distribution is illustrated by real data set. Based on p-values of goodness of fit test, we found that the MGG distribution provides highest p-values when we compared with LGG, GG and three parameters Weibull distributions as shown in Table 3. According to the classical statistics, the MGG distribution is the best fit for these data. In conclusion, it is believed that the MGG distribution may attract wider application in real lifetime data from diverse disciplines. In the future research we should be considered in parameter estimation using Bayesian or other approaches.

\section{ACKNOWLEDGEMENT}

The researchers wish to thank School of Science University of Phayao. Also, the authors thank Dr. Chookait Pudprommarat, the editor and referees for their comments that aided in improving this article

\section{REFERENCES}

Ahmed, A., K.A. Mir and J.A. Reshi, 2013a. On new method of estimation of parameters of size-biased generalized gamma distribution and its structural properties. IOSR J. Mathem., 5: 34-40. DOI: 10.9790/5728-0523440

Ahmed, A., K.A. Mir and J.A. Reshi, 2013b. Structural properties of size-biased gamma distribution. IOSR J. Mathem., 5: 55-61. DOI: 10.9790/5728-0525561

Akinsete, A., F. Famoye and C. Lee, 2008. The betaPareto distribution. Statistics, 42: 547-563. DOI: 10.1080/02331880801983876

Alkarni, S.H., 2012. New family of logarithmic lifetime distributions. J. Math. Stat., 8: 435-440. DOI: 10.3844/jmssp.2012.435.440

Biswas, S.S., B. Alam and M.N. Doja, 2014. A refinement of dijkstraâ $\square{ }^{\mathrm{TM}_{\mathrm{S}}}$ algorithm for extraction of shortest paths in generalized real timemultigraphs. J. Comput. Sci., 10: 593-603. DOI: 10.3844/jcssp.2014.593.603

Cox, C., 2008. The generalized F distribution: An um brella for parametric survival analysis. Stat. Med., 27: 4301-4312. DOI: 10.1002/sim.3292
Cox, C., H. Chu, M.F. Schneider and A. Munoz, 2007. Parametric survival analysis and taxonomy of hazard functions for the generalized gamma distribution. Stat. Med., 26: 4352-4374. DOI: 10.1002/sim. 2836

Cordeiro, G.M., R.R. Pescim and E.M.M. Ortega, 2012. The kumaraswamy generalized half-normal distribution for skewed positive data. J. Data Sci., 10: 195-22.

Mahdi, T. and A.K. Gupta, 2013. A generalization of the gamma distribution. J. Data Sci., 11: 403-414.

Mahesh, C., E. Kannan and M.S. Saravanan, 2014. Generalized regression neural network based expert system for hepatitis b diagnosis. J. Comput. Sci., 10: 563-569. DOI: 10.3844/jessp.2014.563.569

Nadarajah, S. and A.K. Gupta, 2007. A generalized gamma distribution with application to drought data. Mathem. Comput. Simulation, 74: 1-7. DOI: 10.1016/j.matcom.2006.04.004

Nanuwong, N. and W. Bodhisuwan, 2014. Length biased beta-pareto distribution and its structural properties with application. J. Math. Stat., 10: 49-57. DOI: 10.3844/jmssp.2014.49.57

Patil, G.P. and C.R. Rao, 1978. Weighted distributions and size-biased sampling with applications to wildlife populations and human families. Biometrics, 34: 179-189. DOI: 10.2307/2530008

Patill, G.P., C.R. Rao and M.V. Ratnaparkhi, 1986. On discrete weighted distributions and their use in model choice for observed data. Commun. StatisitTheory Math., 15: 907-918. DOI: 10.1080/03610928608829159

Resti, Y., N. Ismail and S.H. Jamaan, 2013. Estimation of claim cost data using zero adjusted gamma and inverse Gaussian regression models. J. Math. Stat., 9: 186-192. DOI: 10.3844/jmssp.2013.186.192

Sattayatham, P. and T. Talangtam, 2012. Fitting of finite mixture distributions to motor insurance claims. J. Math. Stat. 8 : 49-56. DOI: 10.3844/jmssp.2012.49.56

Stacy, E.W., 1962. A generalization of the gamma distri bution. Annals Mathem. Statist., 33: 1187-1192. DOI: $10.1214 /$ aoms/1177704481

Wahyudi, I., I. Purhadi and Sutikno, 2011. The development of parameter estimation on hazard rate of trivariate weibull distribution. Am. J. Biostat., 2: 26-35. DOI: 10.3844/amjbsp.2011.26.350

Kamaruzzaman, Z.A., Z. Isa and M.T. Ismail, 2012. Mixtures of normal distributions: Application to bursa malaysia stock market indices. World Applied Sci. J., 16: 781-790. 